Hossein Aminikhah, Ali Jamalian / TJ MCS Vol .5 No.4 (2012) 241-257

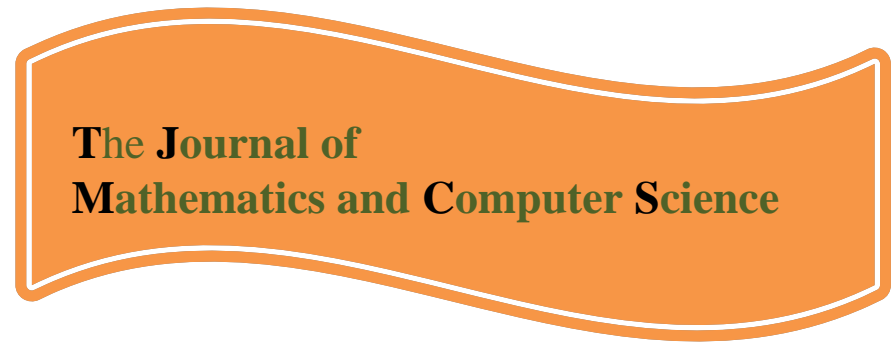

Available online at

http://www.TJMCS.com

The Journal of Mathematics and Computer Science Vol .5 No.4 (2012) 241-257

\title{
An Analytical Approximation for Boundary Layer Flow Convection Heat and Mass Transfer Over a Flat Plate
}

\author{
Hossein Aminikhah \\ Department of Applied Mathematics, School of Mathematical Sciences, University of Guilan, \\ P.O. Box 41335-19141, Rasht, Iran \\ aminikhah@guilan.ac.ir \\ Ali Jamalian \\ Department of Applied Mathematics, School of Mathematical Sciences, University of Guilan, \\ P.O. Box 41335-19141, Rasht, Iran \\ a.jamalain.math.@gmail.com
}

Received: November 2012, Revised: December 2012

Online Publication: December 2012

\begin{abstract}
In this article, Laplace transform and new homotopy perturbation methods are adopted to study the problem of forced convection over a horizontal flat plate analytically. The problem is a system of nonlinear ordinary differential equations which arises in boundary layer flow. The solutions approximated by the proposed method are shown to be precise as compared to the corresponding results obtained by numerical method. A high accuracy of new method is evident.
\end{abstract}

Keywords: Laplace transform; New homotopy perturbation method; Blasius equation.

2010 Mathematics Subject Classification: Primary 35G25; Secondary 65M99. 


\section{Introduction.}

Boundary layer techniques have an important role in developing theory of high-speed flight, separations process, and biological mass transfer systems. Most scientific problems such as mass transfer are nonlinear and do not have analytical solution. One of the well-known equations arising in fluid mechanics and boundary layer approach is Blasius equation [1]. The homotopy perturbation method (HPM) is established by He [2-8] in 1998 to obtain series solutions of nonlinear differential equations. This method has been applied to solve linear and nonlinear equations of heat transfer [9-11], fourth order Volterra integro differential equations [12], boundary value problem [13-14], fluid mechanics [15], nonlinear Schrodinger equations [16], integral equations [17], boundary value problems [18], fractional KdV-Burgers equation [19], nonlinear system of second order boundary value problems [20]. Khan, et al. [21] solved the long porous slider problem by homotopy perturbation method which is coupled nonlinear ordinary differential equations resulting from the momentum equation. Esmaeilpour, D.D. Ganji [22] have used homotopy perturbation method to solve boundary layer flow and convection heat transfer over a flat plate. In this study, we use a different mechanism of transport phenomena and a different approach for solving problem. We construct the solution using a different approach and obtain an analytical approximation to the solution of problem of forced convection over a horizontal flat plate using combination of Laplace transform and new homotopy perturbation method (LTNHPM). The results obtained via LTNHPM are compared with the numerical solutions [23-24] which confirm the validity of the proposed method. LTNHPM has been used for solving Nonlinear Blasius' Viscous Flow Equation [25], Fokker-Plank equation [26] and Fisher type equatins [27] and provides highly accurate numerical solutions for nonlinear differential equations. The rest of the paper is organized as follows: section 2 presents the governing equations of the problem, while homotopy perturbation method and basic idea of LTNHPM are presented in section 3. The application of LTNHPM for the problem and results are described in section 4 . Conclusions are given in section 5 .

\section{Governing equations}

Boundary layer flow over a flat plate is governed by the continuity and the Navier-Stokes equations. The laminar boundary layer on a flat plate in Figure 1 has been a popular system for heat and mass transfer studies. In this system, we give an analysis of forced convection at high mass-transfer rates, and discuss the analogies that hold in this situation. Consider the non-isothermal, steady, twodimentional flow of binary fluid in the system of Figure 1. The fluid properties density, viscosity, specific heat capacity, conductivity and diffusivity are considered constant. Viscous dissipation is 
neglected and there is no homogeneous chemical reactions. The Prandtl boundary layer equations for the laminar region are [28]:

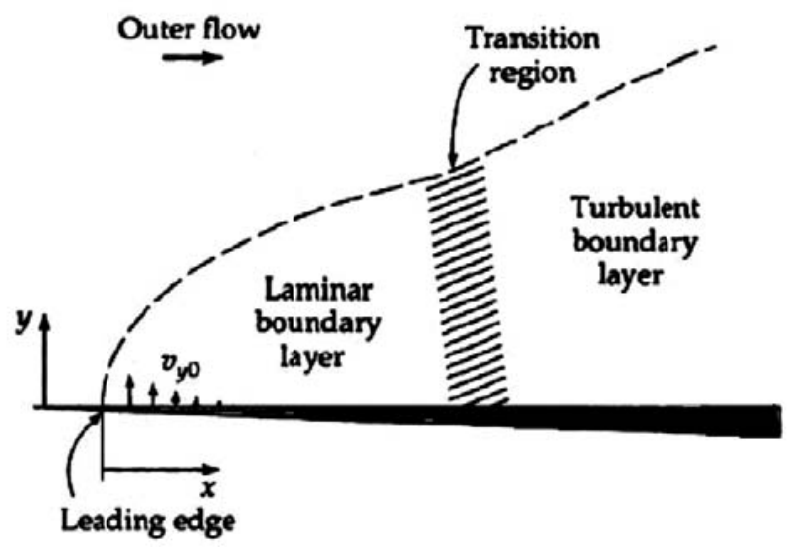

Fig.1. Tangentional flow along a sharp-edge semi infinite flat plate with mass transfer into the stream.

Continuity:

$\frac{\partial u}{\partial x}+\frac{\partial u}{\partial y}=0$

Motion:

$u \frac{\partial u}{\partial x}+v \frac{\partial u}{\partial y}=v \frac{\partial^{2} u}{\partial y^{2}}$

under a boundary layer assumption, the energy equation and continuity of A component are also simplified.

energy:

$u \frac{\partial T}{\partial x}+v \frac{\partial T}{\partial y}=\alpha \frac{\partial^{2} T}{\partial y^{2}}$

continuity of A:

$u \frac{\partial \omega_{A}}{\partial x}+v \frac{\partial \omega_{A}}{\partial y}=D_{A B} \frac{\partial^{2} \omega_{A}}{\partial y^{2}}$

from equations (2), (3) and (4) the solution of the energy-momentum and mass transfermomentum equations are coupled. Thus, in the case of forced convection over a horizontal flat plate, the solution of the momentum equation is decoupled from the energy and continuity component solution. However, the solution of the energy and mass equation are still linked to the momentum solution. The following dimensionless variables are introduced in the transformation:

$\eta=y \sqrt{\frac{U_{\infty}}{v x}}$ 
$\theta=\frac{T-T_{\omega}}{T_{\infty}-T_{\omega}}$

$\Omega=\frac{\omega-\omega_{A o}}{\omega_{\infty}-\omega_{A o}}$

Where $\theta$ and $\Omega$ are non-dimensional form of the temperature and concentration. By equation (1) through equation (7), the governing equations can be reduced to three equations where $f, \theta, \omega$ are functions of the similarity variable $\eta$ :

$$
\left\{\begin{array}{l}
f^{\prime \prime \prime}(\eta)+\frac{1}{2} f(\eta) f^{\prime \prime}(\eta)=0 \\
\theta^{\prime \prime}(\eta)+\frac{\operatorname{Pr}}{2} f(\eta) \theta^{\prime}(\eta)=0 \\
\omega^{\prime \prime}(\eta)+\frac{S c}{2} f(\eta) \omega^{\prime}(\eta)=0
\end{array}\right.
$$

And $f$ is related to the $u$ velocity by $u=U_{\infty} f^{\prime}(\eta)$. The reference velocity is the free stream velocity of force convection. The boundary conditions are:

$\eta=0, \quad f=\frac{d f}{d \eta}=\theta=\omega=0$, and $\eta \rightarrow \infty, \quad \frac{d f}{d \eta}=\theta=\omega=1$.

In 1908, Blasius [1] provided a solution for $f(\eta)$ in the following form

$f(\eta)=\sum_{k=0}^{\infty}\left(-\frac{1}{2}\right) \frac{A_{k} \sigma^{k+1}}{(3 k+2) !} \eta^{3 k+2}$

where $\sigma=f^{\prime \prime}(0), A_{0}=A_{1}=1, A_{k}=\sum_{r=0}^{k-1}\left(\begin{array}{c}3 k-1 \\ 3 r\end{array}\right) A_{r} A_{k-r-1},(k \geq 2)$.

Blasius evaluated $\sigma$ by demonstrating another approximation of $f(\eta)$ at large $\eta$. Then by means of matching two different approximations at a proper point he obtained the numerical result $\sigma=0.332$. In 1938 by means of a numerical technique Howarth [23] gained a more accurate value $\sigma=0.332057$ utilized to solve Blasius equation (7).

\section{Basic idea of LTNHPM}

To illustrate the basic ideas of this method, let us consider the following nonlinear differential equation

$A(u)-f(r)=0, \quad r \in \Omega$,

with the following initial conditions

$u(0)=\alpha_{0}, u^{\prime}(0)=\alpha_{1}, \ldots, u^{(n-1)}(0)=\alpha_{n-1}$

where $A$ is a general differential operator and $f(r)$ is a known analytical function. The operator $A$ can be divided into two parts, $L$ and $N$, where $L$ is a linear and $N$ is a nonlinear operator. Therefore, (10) can be rewritten as 
$L(u)+N(u)-f(r)=0$

Based on NHPM [25], we construct a homotopy $U(r, p): \Omega \times[0,1] \rightarrow \mathrm{R}$, which satisfies

$H(U, p)=(1-p)\left[L(U)-u_{0}\right]+p[A(U)-f(r)]=0, p \in[0,1], r \in \Omega$,

or equivalently,

$H(U, p)=L(U)-u_{0}+p u_{0}+p[N(U)-f(r)]=0$,

where $p \in[0,1]$ is an embedding parameter, $u_{0}$ is an initial approximation for the solution of $(10)$.

Clearly, (13) and (14) give

$H(U, 0)=L(U)-u_{0}=0$,

$H(U(x), 1)=A(U)-f(r)=0$.

Applying Laplace transform to the both sides of (14), we arrive at

$\mathcal{L}\left\{L(U)-u_{0}+p u_{0}+p[N(U)-f(r)]\right\}=0$

Using the differential property of Laplace transform we have

$s^{n} \mathcal{L}\{U\}-s^{n-1} U(0)-s^{n-2} U^{\prime}(0)-\cdots-U^{(n-1)}(0)=\mathcal{L}\left\{u_{0}-p u_{0}+p[N(U)-f(r)]\right\}$

or

$\mathcal{L}\{U\}=\frac{1}{s^{n}}\left\{s^{n-1} U(0)+s^{n-2} U^{\prime}(0)+\cdots+U^{(n-1)}(0)+\mathcal{L}\left\{u_{0}-p u_{0}+p[N(U)-f(r)]\right\}\right\}$

Finally, applying the inverse Laplace transform to the both sides of (19), one can successfully reach to the following

$U=\mathcal{L}^{-1}\left\{\frac{1}{s^{n}}\left\{s^{n-1} U(0)+s^{n-2} U^{\prime}(0)+\cdots+U^{(n-1)}(0)+\mathcal{L}\left\{u_{0}-p u_{0}+p[N(U)-f(r)]\right\}\right\}\right\}$

According to the HPM, we can first use the embedding parameter $p$ as a small parameter, and assume that the solutions of (20) can be represented as a power series in $p$ as

$U(x)=\sum_{n=0}^{\infty} p^{n} U_{n}$.

Now let us rewrite (20) using (21) as

$$
\begin{gathered}
\sum_{n=0}^{\infty} p^{n} U_{n}=\mathcal{L}^{-1}\left\{\frac { 1 } { s ^ { n } } \left\{s^{n-1} U(0)+s^{n-2} U^{\prime}(0)+\cdots+U^{(n-1)}(0)\right.\right. \\
\left.\left.+\mathcal{L}^{-1}\left\{u_{0}-p u_{0}+p\left[N\left(\sum_{n=0}^{\infty} p^{n} U_{n}\right)-f(r)\right]\right\}\right\}\right\}
\end{gathered}
$$

Therefore, equating the coefficients of $p$ with the same power leads to 


$$
\begin{aligned}
& p^{0}: U_{0}=\mathcal{L}^{-1}\left\{\frac{1}{s^{n}}\left(s^{n-1} U(0)+s^{n-2} U^{\prime}(0)+\cdots+U^{(n-1)}(0)+\mathrm{L}\left\{u_{0}\right\}\right)\right\}, \\
& p^{1}: U_{1}=\mathcal{L}^{-1}\left\{\frac{1}{s^{n}}\left(\mathrm{~L}\left\{N\left(U_{0}\right)-u_{0}-f(r)\right\}\right)\right\}, \\
& p^{2}: U_{2}=\mathcal{L}^{-1}\left\{\frac{1}{s^{n}}\left(\mathrm{~L}\left\{N\left(U_{0}, U_{1}\right)\right\}\right)\right\}, \\
& p^{3}: U_{3}=\mathcal{L}^{-1}\left\{\frac{1}{s^{n}}\left(\mathrm{~L}\left\{N\left(U_{0}, U_{1}, U_{2}\right)\right\}\right)\right\}, \\
& \vdots \\
& p^{j}: U_{j}=\mathcal{L}^{-1}\left\{\frac{1}{s^{n}}\left(\mathrm{~L}\left\{N\left(U_{0}, U_{1}, U_{2}, \ldots, U_{j-1}\right)\right\}\right)\right\},
\end{aligned}
$$

Suppose that the initial approximation has the form $U(0)=u_{0}=\alpha_{0}, U^{\prime}(0)=\alpha_{1}, \ldots, U^{(n-1)}(0)=\alpha_{n-1}$, therefore the exact solution may be obtained as following $u=\lim _{p \rightarrow 1} U=U_{0}+U_{1}+U_{2}+\cdots$

\section{Method of solution}

Consider the system of nonlinear ordinary differential equations (8). For solving this nonlinear system by applying the new homotopy perturbation method, we construct the following homotopy

$$
\left\{\begin{array}{l}
H(F(\eta), p)=F^{\prime \prime \prime}(\eta)-f_{0}(\eta)+p\left[f_{0}(\eta)+\frac{1}{2} F(\eta) F^{\prime \prime}(\eta)\right]=0, \\
H(\Theta(\eta), p)=\Theta^{\prime \prime}(\eta)-\theta_{0}(\eta)+p\left[\theta_{0}(\eta)+\frac{\operatorname{Pr}}{2} F(\eta) \Theta^{\prime}(\eta)\right]=0, \\
H(\Omega(\eta), p)=\Omega^{\prime \prime}(\eta)-\omega_{0}(\eta)+p\left[\omega_{0}(\eta)+\frac{S c}{2} F(\eta) \Omega^{\prime}(\eta)\right]=0,
\end{array}\right.
$$

where $p \in[0,1]$ is an embedding parameter, $f_{0}(\eta), \theta_{0}(\eta), \omega_{0}(\eta)$ are initial approximations of solution of system (8). Clearly, we have from system of equations (25):

$$
\left\{\begin{array}{l}
H(F(\eta), 0)=F^{\prime \prime \prime}(\eta)-f_{0}(\eta)=0, \\
H(\Theta(\eta), 0)=\Theta^{\prime \prime}(\eta)-\theta_{0}(\eta)=0, \\
H(\Omega(\eta), 0)=\Omega^{\prime \prime}(\eta)-\omega_{0}(\eta)=0,
\end{array}\right.
$$

and

$$
\left\{\begin{array}{l}
H(F(\eta), 1)=F^{\prime \prime \prime}(\eta)+\frac{1}{2} F(\eta) F^{\prime \prime}(\eta)=0, \\
H(\Theta(\eta), 1)=\Theta^{\prime \prime}(\eta)+\frac{\operatorname{Pr}}{2} F(\eta) \Theta^{\prime}(\eta)=0, \\
H(\Omega(\eta), 1)=\Omega^{\prime \prime}(\eta)+\frac{S c}{2} F(\eta) \Omega^{\prime}(\eta)=0,
\end{array}\right.
$$

By applying Laplace transform on both sides of (25), we have 


$$
\left\{\begin{array}{l}
\mathcal{L}\left\{F^{\prime \prime \prime}(\eta)-f_{0}(\eta)+p\left[f_{0}(\eta)+\frac{1}{2} F(\eta) F^{\prime \prime}(\eta)\right]\right\}=0, \\
\mathcal{L}\left\{\Theta^{\prime \prime}(\eta)-\theta_{0}(\eta)+p\left[\theta_{0}(\eta)+\frac{\operatorname{Pr}}{2} F(\eta) \Theta^{\prime}(\eta)\right]\right\}=0, \\
\mathcal{L}\left\{\Omega^{\prime \prime}(\eta)-\omega_{0}(\eta)+p\left[\omega_{0}(\eta)+\frac{S C}{2} F(\eta) \Omega^{\prime}(\eta)\right]\right\}=0,
\end{array}\right.
$$

Using the differential property of Laplace transform we have

$$
\left\{\begin{array}{l}
s^{3} \mathcal{L}\{F(\eta)\}-s^{2} F(0)-s F^{\prime}(0)-F^{\prime \prime}(0)=\mathcal{L}\left\{f_{0}(\eta)-p\left[f_{0}(\eta)+\frac{1}{2} F(\eta) F^{\prime \prime}(\eta)\right]\right\} \\
s^{2} \mathcal{L}\{\Theta(\eta)\}-s \Theta(0)-\Theta^{\prime}(0)=\mathcal{L}\left\{\theta_{0}(\eta)-p\left[\theta_{0}(\eta)+\frac{\operatorname{Pr}}{2} F(\eta) \Theta^{\prime}(\eta)\right]\right\} \\
s^{2} \mathcal{L}\{\Omega(\eta)\}-s \Omega(0)-\Omega^{\prime}(0)=\mathcal{L}\left\{\omega_{0}(\eta)-p\left[\omega_{0}(\eta)+\frac{S c}{2} F(\eta) \Omega^{\prime}(\eta)\right]\right\}
\end{array}\right.
$$

or

$$
\left\{\begin{array}{l}
\mathcal{L}\{F(\eta)\}=\frac{1}{s^{3}}\left(s^{2} F(0)+s F^{\prime}(0)+F^{\prime \prime}(0)+\mathcal{L}\left\{f_{0}(\eta)-p\left[f_{0}(\eta)+\frac{1}{2} F(\eta) F^{\prime \prime}(\eta)\right]\right\}\right) \\
\mathcal{L}\{\Theta(\eta)\}=\frac{1}{s^{2}}\left(s \Theta(0)+\Theta^{\prime}(0)+\mathcal{L}\left\{\theta_{0}(\eta)-p\left[\theta_{0}(\eta)+\frac{\operatorname{Pr}}{2} F(\eta) \Theta^{\prime}(\eta)\right]\right\}\right), \\
\mathcal{L}\{\Omega(\eta)\}=\frac{1}{s^{2}}\left(s \Omega(0)+\Omega^{\prime}(0)+\mathcal{L}\left\{\omega_{0}(\eta)-p\left[\omega_{0}(\eta)+\frac{S c}{2} F(\eta) \Omega^{\prime}(\eta)\right]\right\}\right),
\end{array}\right.
$$

By applying inverse Laplace transform on both sides of (30), we have

$$
\left\{\begin{array}{l}
F(\eta)=\mathcal{L}^{-1}\left\{\frac{1}{s^{3}}\left(s^{2} F(0)+s F^{\prime}(0)+F^{\prime \prime}(0)+\mathcal{L}\left\{f_{0}(\eta)-p\left[f_{0}(\eta)+\frac{1}{2} F(\eta) F^{\prime \prime}(\eta)\right]\right\}\right),\right. \\
\Theta(\eta)=\mathcal{L}^{-1}\left\{\frac{1}{s^{2}}\left(s \Theta(0)+\Theta^{\prime}(0)+\mathcal{L}\left\{\theta_{0}(\eta)-p\left[\theta_{0}(\eta)+\frac{\operatorname{Pr}}{2} F(\eta) \Theta^{\prime}(\eta)\right]\right\}\right)\right\} \\
\Omega(\eta)=\mathcal{L}^{-1}\left\{\frac{1}{s^{2}}\left(s \Omega(0)+\Omega^{\prime}(0)+\mathcal{L}\left\{\omega_{0}(\eta)-p\left[\omega_{0}(\eta)+\frac{S c}{2} F(\eta) \Omega^{\prime}(\eta)\right]\right\}\right)\right\}
\end{array}\right.
$$

According to the HPM, we use the embedding parameter $p$ as a small parameter, and assume that the solutions of equation (30) can be represented as a power series in $p$ as

$$
\left\{\begin{array}{l}
F(\eta)=\sum_{n=0}^{\infty} p^{n} F_{n}(\eta), \\
\Theta(\eta)=\sum_{n=0}^{\infty} p^{n} \Theta_{n}(\eta), \\
\Omega(\eta)=\sum_{n=0}^{\infty} p^{n} \Omega_{n}(\eta),
\end{array}\right.
$$

Substituting (32) into (31), and equating the terms with the identical powers of $p$, leads to 


$$
\begin{aligned}
& p^{0}:\left\{\begin{array}{l}
F_{0}(\eta)=\mathcal{L}^{-1}\left\{\frac{F(0)}{s}+\frac{F^{\prime}(0)}{s^{2}}+\frac{F^{\prime \prime}(0)}{s^{3}}+\mathcal{L}\left\{f_{0}(\eta)\right\}\right\} \\
\Theta_{0}(\eta)=\mathcal{L}^{-1}\left\{\frac{\Theta(0)}{s}+\frac{\Theta^{\prime}(0)}{s^{2}}+\mathcal{L}\left\{\theta_{0}(\eta)\right\}\right\} \\
\Omega_{0}(\eta)=\mathcal{L}^{-1}\left\{\frac{\Omega(0)}{s}+\frac{\Omega^{\prime}(0)}{s^{2}}+\mathcal{L}\left\{\omega_{0}(\eta)\right\}\right\}
\end{array}\right. \\
& p^{1}:\left\{\begin{array}{l}
F_{1}(\eta)=\mathcal{L}^{-1}\left\{-\frac{1}{s^{3}} \mathcal{L}\left\{f_{0}(\eta)+\frac{1}{2} F_{0}(\eta) F_{0}^{\prime \prime}(\eta)\right\}\right\}, \\
\Omega_{1}(\eta)=\mathcal{L}^{-1}\left\{-\frac{1}{s^{2}} \mathcal{L}\left\{\theta_{0}(\eta)+\frac{\operatorname{Pr}}{2} F_{0}(\eta) \Theta_{0}^{\prime}(\eta)\right\}\right\},
\end{array}\right. \\
& p^{2}:\left\{\begin{array}{l}
F_{2}(\eta)=\mathcal{L}^{-1}\left\{-\frac{1}{2 s^{3}} \mathcal{L}\left\{F_{0}(\eta) F_{1}^{\prime \prime}(\eta)+F_{1}(\eta) F_{0}^{\prime \prime}(\eta)\right\}\right\}, \\
\Theta_{2}(\eta)=\mathcal{L}^{-1}\left\{-\frac{\operatorname{Pr}}{2 s^{2}} \mathcal{L}\left\{F_{0}(\eta) \Theta_{1}^{\prime}(\eta)+F_{1}(\eta) \Theta_{0}^{\prime}(\eta)\right\}\right\}, \\
\Omega_{2}(\eta)=\mathcal{L}^{-1}\left\{-\frac{S c}{2 s^{2}} \mathcal{L}\left\{F_{0}(\eta) \Omega_{1}^{\prime}(\eta)+F_{1}(\eta) \Omega_{0}^{\prime}(\eta)\right\}\right\},
\end{array}\right. \\
& p^{3}:\left\{\begin{array}{l}
F_{3}(\eta)=\mathcal{L}^{-1}\left\{-\frac{1}{2 s^{3}} \mathcal{L}\left\{F_{0}(\eta) F_{2}^{\prime \prime}(\eta)+F_{1}(\eta) F_{1}^{\prime \prime}(\eta)+F_{2}(\eta) F_{0}^{\prime \prime}(\eta)\right\}\right\}, \\
\Theta_{3}(\eta)=\mathcal{L}^{-1}\left\{-\frac{\operatorname{Pr}}{2 s^{2}} \mathcal{L}\left\{F_{0}(\eta) \Theta_{2}^{\prime}(\eta)+F_{1}(\eta) \Theta_{1}^{\prime}(\eta)+F_{2}(\eta) \Theta_{0}^{\prime}(\eta)\right\}\right\}, \\
\Omega_{3}(\eta)=\mathcal{L}^{-1}\left\{-\frac{S c}{2 s^{2}} \mathcal{L}\left\{F_{0}(\eta) \Omega_{2}^{\prime}(\eta)+F_{1}(\eta) \Omega_{1}^{\prime}(\eta)+F_{2}(\eta) \Omega_{0}^{\prime}(\eta)\right\}\right\},
\end{array}\right. \\
& \text { : } \\
& p^{j}:\left\{\begin{array}{l}
F_{j}(\eta)=\mathcal{L}^{-1}\left\{-\frac{1}{2 s^{3}} \mathcal{L}\left\{\sum_{k=0}^{j-1} F_{k}(\eta) F_{j-k-1}^{\prime \prime}(\eta)\right\}\right\}, \\
\mathcal{L}^{-1}\left\{-\frac{\operatorname{Pr}}{2 s^{2}} \mathcal{L}\left\{\sum_{k=0}^{j-1} F_{k}(\eta) \Theta_{j-k-1}^{\prime}\right\}\right\}, \\
\Omega_{j}(\eta)=\mathcal{L}^{-1}\left\{-\frac{S c}{2 s^{2}} \mathcal{L}\left\{\sum_{k=0}^{j-1} F_{k}(\eta) \Omega_{j-k-1}^{\prime}\right\}\right\},
\end{array}\right.
\end{aligned}
$$

To complete the solution we choose $f_{0}(\eta)=F(0)=f(0)=0, F^{\prime}(0)=f^{\prime}(0)=0$ and $F^{\prime \prime}(0)=f^{\prime \prime}(0)=\sigma$. Note that by replacing $\theta$ with $f^{\prime}$ and choosing $\operatorname{Pr}=1$, so that the energy equation and momentum equation are equivalent. So we choose $\theta_{0}(\eta)=\omega_{0}(\eta)=\Theta(0)=\Omega(0)=\theta(0)=\omega(0)=0$ and $\Theta^{\prime}(0)=\theta^{\prime}(0)=\Omega^{\prime}(0)=\omega^{\prime}(0)=\sigma$. Solving the equation (33) for $F_{j}(\eta), \Theta_{j}(\eta), \Omega_{j}(\eta), j=0,1, \cdots$ leads to the results 


$$
\begin{aligned}
& \left\{\begin{array}{l}
F_{0}(\eta)=\frac{1}{2} \sigma \eta^{2} \\
\Theta_{0}(\eta)=\sigma \eta \\
\Omega_{0}(\eta)=\sigma \eta
\end{array},\right. \\
& \left\{\begin{array}{l}
F_{1}(\eta)=-\frac{1}{240} \sigma^{2} \eta^{5} \\
\Theta_{1}(\eta)=-\frac{\operatorname{Pr}}{48} \sigma^{2} \eta^{4} \\
\Omega_{1}(\eta)=-\frac{S c}{48} \sigma^{2} \eta^{4}
\end{array}\right. \\
& \left\{\begin{array}{l}
F_{2}(\eta)=\frac{11}{161280} \sigma^{3} \eta^{8} \\
\Theta_{2}(\eta)=\frac{\operatorname{Pr}}{20160} \sigma^{3}(1+10 \operatorname{Pr}) \eta^{7} \\
\Omega_{2}(\eta)=\frac{S c}{20160} \sigma^{3}(1+10 S c) \eta^{7}
\end{array}\right. \\
& \left\{\begin{array}{l}
F_{3}(\eta)=-\frac{5}{4257792} \sigma^{4} \eta^{11} \\
\Theta_{3}(\eta)=-\frac{\operatorname{Pr}}{29030400} \sigma^{4}\left(11+84 \mathrm{Pr}+280 \mathrm{Pr}^{2}\right) \eta^{10}, \\
\Omega_{3}(\eta)=-\frac{S c}{29030400} \sigma^{4}\left(11+84 S c+280 S c^{2}\right) \eta^{10}
\end{array}\right. \\
& \left\{\begin{array}{l}
F_{4}(\eta)=\frac{9299}{464950886400} \sigma^{5} \eta^{14} \\
\Theta_{4}(\eta)=\frac{\operatorname{Pr}}{99632332800} \sigma^{5}\left(375+2882 \mathrm{Pr}+9240 \mathrm{Pr}^{2}+15400 \mathrm{Pr}^{3}\right) \eta^{13}, \\
\Omega_{4}(\eta)=\frac{S c}{99632332800} \sigma^{5}\left(375+2882 \mathrm{Sc}+9240 \mathrm{Sc}^{2}+15400 \mathrm{Sc}^{3}\right) \eta^{13}
\end{array}\right. \\
& \left\{\begin{array}{l}
F_{5}(\eta)=-\frac{127239}{3793999233024000} \sigma^{6} \eta \\
\Theta_{5}(\eta)=-\frac{\operatorname{Pr}}{183936614400} \sigma^{6}\left(27897+225680 \mathrm{Pr}+76076 \mathrm{Pr}^{2}+140140 \mathrm{Pr}^{3}+140140 \mathrm{Pr}^{4}\right) \eta^{16} \\
\Omega_{5}(\eta)=-\frac{S c}{183936614400} \sigma^{6}\left(27897+225680 \mathrm{Sc}+76076 \mathrm{Sc}^{2}+140140 \mathrm{Sc}^{3}+140140 \mathrm{Sc}^{4}\right) \eta^{16}
\end{array}\right.
\end{aligned}
$$

Therefore we gain the solution of equation (8) as

$$
\begin{aligned}
f(\eta) & =\lim _{p \rightarrow 1} \sum_{n=0}^{\infty} p^{n} F_{n}(\eta)= \\
& =F_{0}(\eta)+F_{1}(\eta)+F_{2}(\eta)+\cdots \\
& =\frac{1}{2} \sigma \eta^{2}-\frac{1}{240} \sigma^{2} \eta^{5}+\frac{11}{161280} \sigma^{3} \eta^{8}-\frac{5}{4257792} \sigma^{4} \eta^{11}+\cdots
\end{aligned}
$$




$$
\begin{aligned}
\theta(\eta) & =\lim _{p \rightarrow 1} \sum_{n=0}^{\infty} p^{n} \Theta_{n}(\eta)= \\
& =\Theta_{0}(\eta)+\Theta_{1}(\eta)+\Theta_{2}(\eta)+\cdots \\
& =\sigma \eta-\frac{\operatorname{Pr}}{48} \sigma^{2} \eta^{4}+\frac{\operatorname{Pr}}{20160} \sigma^{3}(1+10 \operatorname{Pr}) \eta^{7}-\frac{\operatorname{Pr}}{29030400} \sigma^{4}\left(11+84 \operatorname{Pr}+280 \operatorname{Pr}^{2}\right) \eta^{10}+\cdots \\
\omega(\eta) & =\lim _{p \rightarrow 1} \sum_{n=0}^{\infty} p^{n} \Omega_{n}(\eta)= \\
& =\Omega_{0}(\eta)+\Omega_{1}(\eta)+\Omega_{2}(\eta)+\cdots \\
& =\sigma \eta-\frac{S c}{48} \sigma^{2} \eta^{4}+\frac{S c}{20160} \sigma^{3}(1+10 S c) \eta^{7}-\frac{S c}{29030400} \sigma^{4}\left(11+84 S c+280 S c^{2}\right) \eta^{10}+\cdots
\end{aligned}
$$

Inserting $\sigma=0.332057, \operatorname{Pr}=1, S c=1$, therefore, the analytical approximation to the solution can be expressed as

$$
\begin{aligned}
f(\eta) & =0.1660285000 \eta^{2}-0.0004594243800 \eta^{5}+0.000002497181392 \eta^{8} \\
& -1.427697248 \times 10^{-8} \eta^{11}+8.074067341 \times 10^{-11} \eta^{14}-4.495676921 \times 10^{-13} \eta^{17} \\
& +2.475361576 \times 10^{-15} \eta^{20}-1.353816100 \times 10^{-17} \eta^{23}+7.376815264 \times 10^{-20} \eta^{26} \\
& -4.011691315 \times 10^{-22} \eta^{29}+2.179432978 \times 10^{-24} \eta^{32}+\cdots \\
\theta(\eta) & =0.332057 \eta-0.2297121900 \times 10^{-2} \eta^{4}+0.1997745114 \times 10^{-4} \eta^{7} \\
& -1.570466972 \times 10^{-7} \eta^{10}+1.130369428 \times 10^{-9} \eta^{13}-7.642650764 \times 10^{-12} \eta^{16} \\
& +4.950723152 \times 10^{-14} \eta^{19}-3.113777029 \times 10^{-16} \eta^{22}+1.917971969 \times 10^{-18} \eta^{25} \\
& -1.163390481 \times 10^{-20} \eta^{28}+6.974185531 \times 10^{-23} \eta^{31}+\cdots
\end{aligned}
$$

and

$$
\begin{aligned}
\omega(\eta) & =0.332057 \eta-0.2297121900 \times 10^{-2} \eta^{4}+0.1997745114 \times 10^{-4} \eta^{7} \\
& -1.570466972 \times 10^{-7} \eta^{10}+1.130369428 \times 10^{-9} \eta^{13}-7.642650764 \times 10^{-12} \eta^{16} \\
& +4.950723152 \times 10^{-14} \eta^{19}-3.069070891 \times 10^{-16} \eta^{22}+1.904364065 \times 10^{-18} \eta^{25} \\
& -1.156666375 \times 10^{-20} \eta^{28}+6.935771995 \times 10^{-23} \eta^{31}+\cdots
\end{aligned}
$$

Suppose, $\quad f^{* *}(\eta)=\sum_{n=0}^{10} F_{n}(\eta), f^{*}(\eta)=\sum_{n=0}^{6} F_{n}(\eta), g^{* *}(\eta)=\frac{d}{d \eta} f^{* *}(\eta) \quad$ and $\quad g^{*}(\eta)=\frac{d}{d \eta} f^{*}(\eta), \quad$ some numerical results of these solutions are presented in Table 1, Table 2, Figure 1 and 2.

Table 1 is made to compare between present results and results given by Howarth [23] for $f(\eta)$. In Fig. 1, Fig. 2 one can also see the comparison between LTNHPM results and Howarth's results. 
Hossein Aminikhah, Ali Jamalian / TJ MCS Vol .5 No.4 (2012) 241-257

Table 1: comparison between Numerical method and LTNHPM method for $f(\eta)$

\begin{tabular}{cccc}
\hline$\eta$ & Hawrth $[23]$ & $f^{*}(\eta)$ & $f^{* *}(\eta)$ \\
\hline 0 & 0.00000 & 0.00000 & 0.00000 \\
0.2 & 0.00664 & 0.00664 & 0.00664 \\
0.4 & 0.02656 & 0.02656 & 0.02656 \\
0.6 & 0.05974 & 0.05973 & 0.05973 \\
0.8 & 0.10611 & 0.10611 & 0.10611 \\
1 & 0.16557 & 0.16557 & 0.16557 \\
1.2 & 0.23795 & 0.23795 & 0.23795 \\
1.4 & 0.32298 & 0.32298 & 0.32298 \\
1.6 & 0.42032 & 0.42032 & 0.42032 \\
1.8 & 0.52952 & 0.52952 & 0.52952 \\
2 & 0.65003 & 0.65002 & 0.65002 \\
2.2 & 0.78120 & 0.78119 & 0.78119 \\
2.4 & 0.92230 & 0.92228 & 0.92228 \\
2.6 & 1.07252 & 1.07250 & 1.07250 \\
2.8 & 1.23099 & 1.23098 & 1.23098 \\
3 & 1.39682 & 1.39681 & 1.39681 \\
3.2 & 1.56911 & 1.56909 & 1.56909 \\
3.4 & 1.74696 & 1.74697 & 1.74695 \\
3.6 & 1.92954 & 1.92959 & 1.92952 \\
3.8 & 2.11605 & 2.11625 & 2.11602 \\
4 & 2.30576 & 2.30645 & 2.30575 \\
4.2 & 2.49806 & 2.50012 & 2.49809 \\
4.4 & 2.69238 & 2.69818 & 2.69263 \\
4.6 & 2.88826 & 2.90374 & 2.88946 \\
4.8 & 3.08534 & 3.12469 & 3.09047 \\
5 & 3.28239 & 3.37923 & 3.30378 \\
\hline & & &
\end{tabular}

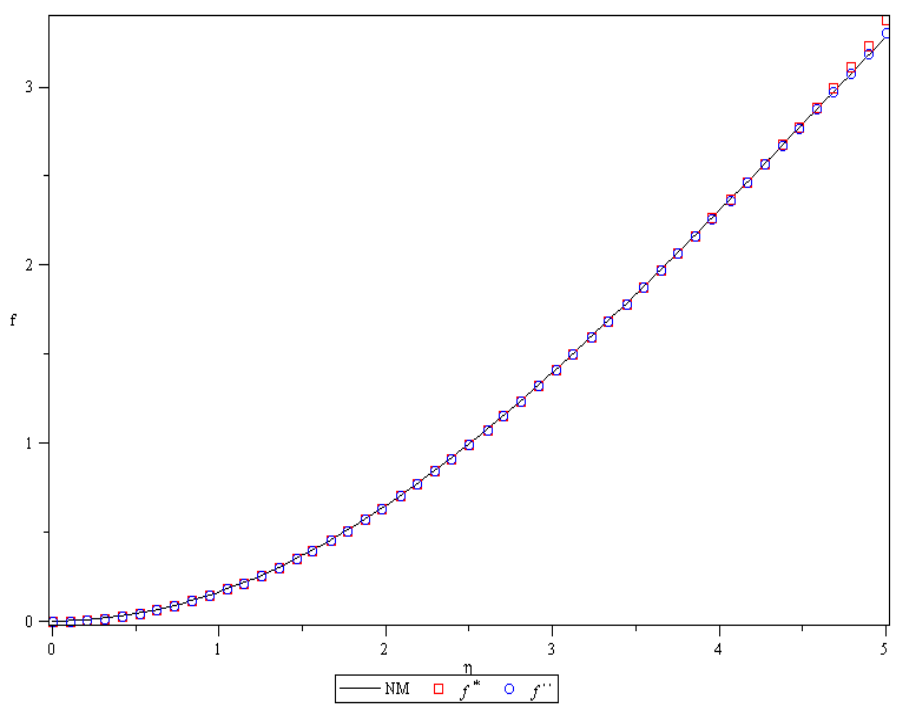

Fig.1 The comparison of answers obtained by LTNHPM and Numerical method results for $f(\eta)$ 
Hossein Aminikhah, Ali Jamalian / TJ MCS Vol .5 No.4 (2012) 241-257

Table 2: comparison between numerical and LTNHPM methods for $f^{\prime}(\eta)$

\begin{tabular}{cccc}
\hline$\eta$ & Hawrth $[23]$ & $g^{*}(\eta)$ & $g^{* *}(\eta)$ \\
\hline 0 & 0.00000 & 0.00000 & 0.00000 \\
0.2 & 0.06641 & 0.06641 & 0.06641 \\
0.4 & 0.13277 & 0.13276 & 0.13276 \\
0.6 & 0.19894 & 0.19894 & 0.19894 \\
0.8 & 0.26471 & 0.26471 & 0.26471 \\
1 & 0.32979 & 0.32978 & 0.32978 \\
1.2 & 0.39378 & 0.39378 & 0.39378 \\
1.4 & 0.45627 & 0.45626 & 0.45626 \\
1.6 & 0.51676 & 0.51676 & 0.51676 \\
1.8 & 0.57477 & 0.57476 & 0.57476 \\
2 & 0.62977 & 0.62977 & 0.62977 \\
2.2 & 0.68132 & 0.68131 & 0.68131 \\
2.4 & 0.72899 & 0.72898 & 0.72898 \\
2.6 & 0.77246 & 0.77245 & 0.77245 \\
2.8 & 0.81152 & 0.81151 & 0.81151 \\
3 & 0.84605 & 0.84605 & 0.84604 \\
3.2 & 0.87609 & 0.87611 & 0.87608 \\
3.4 & 0.90177 & 0.90188 & 0.90176 \\
3.6 & 0.92333 & 0.92375 & 0.92333 \\
3.8 & 0.94112 & 0.94244 & 0.94113 \\
4 & 0.95552 & 0.95944 & 0.95560 \\
4.2 & 0.96696 & 0.97794 & 0.96740 \\
4.4 & 0.97587 & 1.00506 & 0.97795 \\
4.6 & 0.98269 & 1.05663 & 0.99167 \\
4.8 & 0.98779 & 1.16716 & 1.02418 \\
5 & 0.99155 & 1.40961 & 1.13019 \\
\hline & & &
\end{tabular}

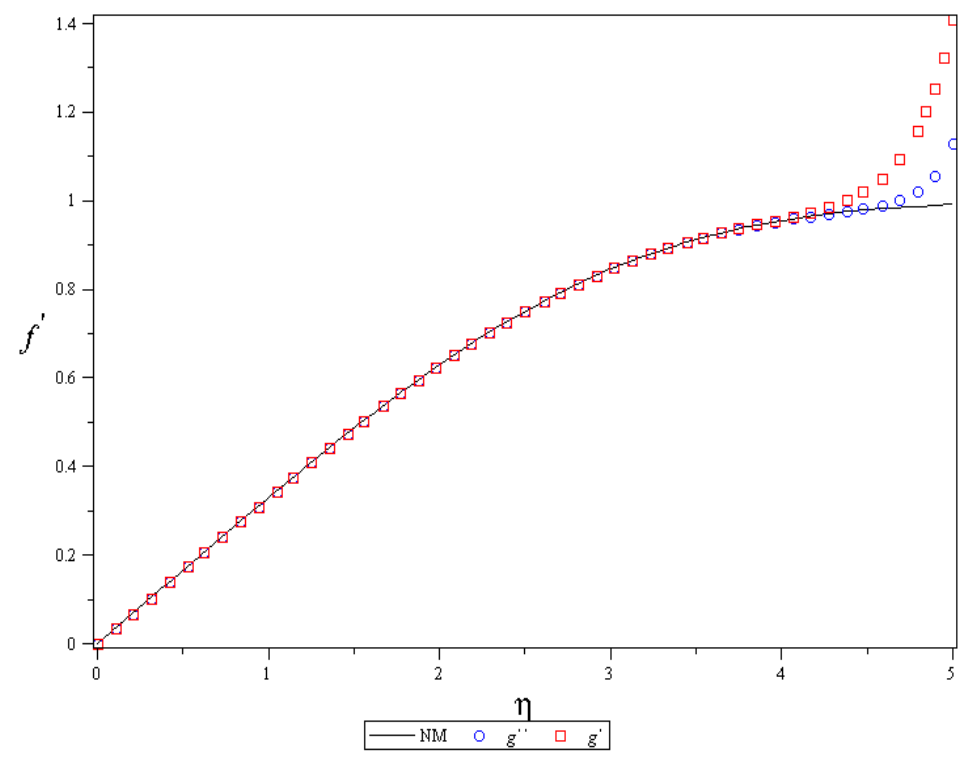

Fig.2 The comparison of answers obtained by LTNHPM and Numerical method results for $f^{\prime}(\eta)$ 
Hossein Aminikhah, Ali Jamalian / TJ MCS Vol .5 No.4 (2012) 241-257

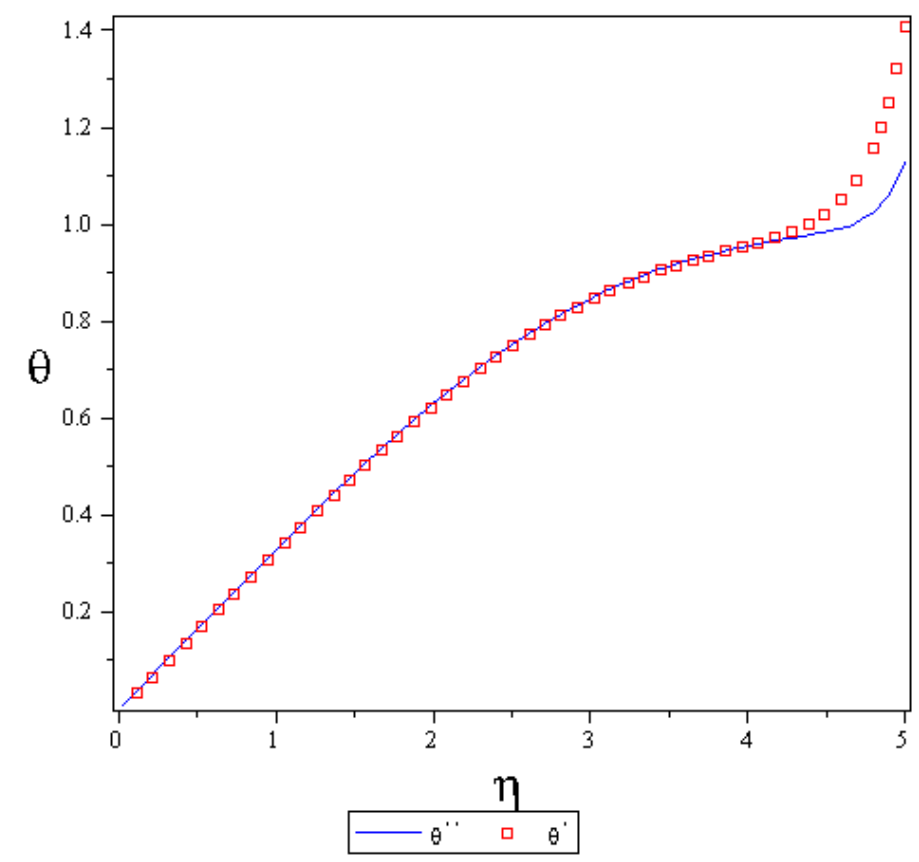

Fig.3 The comparison of 6-terms and 10-terms for $\theta(\eta), \operatorname{Pr}=1$ obtained by LTNHPM.

With assumption $\operatorname{Pr}=S c=1, \theta(\eta)$ and $\omega(\eta)$ are equivalent (figure 3). Results for different values of Prandtl number and Schmidt number has been shown in figure 4 and figure 5.

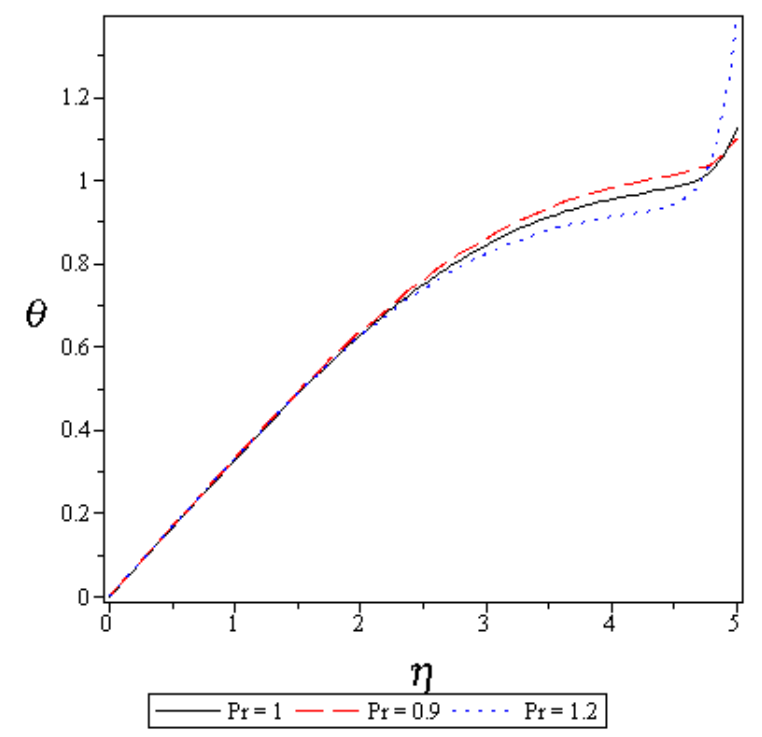

Fig.4 The comparison of 10-terms for $\theta(\eta)$ obtained by LTNHPM with different Prandtl numbers. 


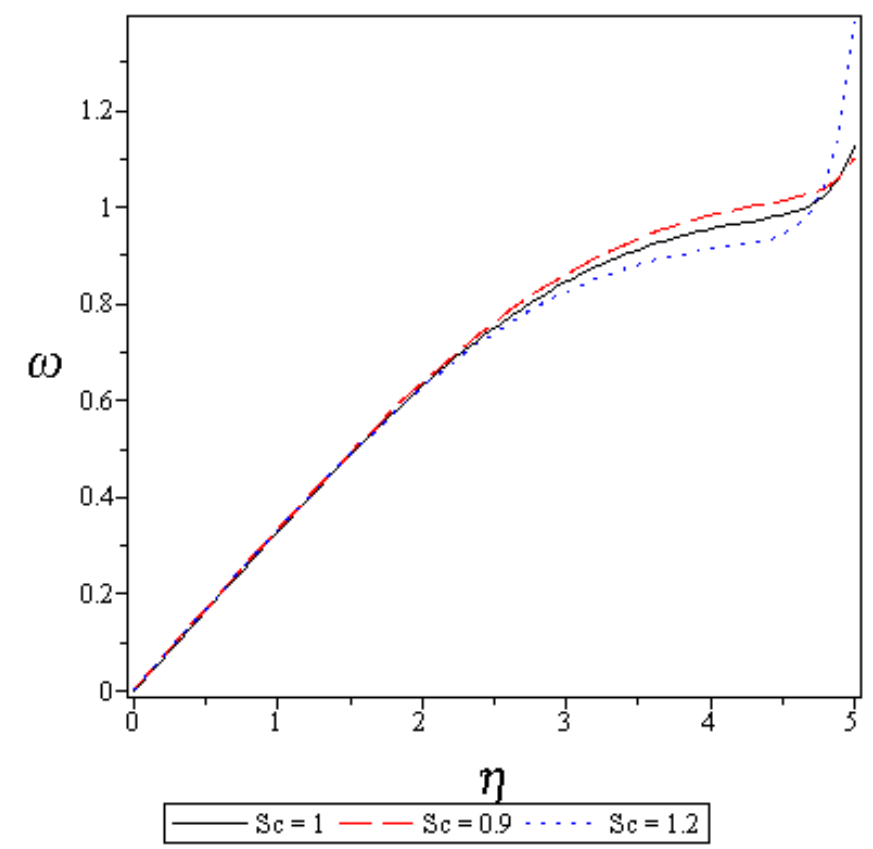

Fig.5 The comparison of 10-terms for $\omega(\eta)$ obtained by LTNHPM with different Schmidt numbers.

\section{Conclusion}

In this paper, the combined Laplace transform and homotopy perturbation methods is employed to give numerical solutions of nonlinear system, the problem of forced convection over a horizontal flat. To illustrate the accuracy and efficiency of the proposed procedure, various different examples in the interval $0 \leq \eta \leq 5$ have also been analyzed and the numerical results are listed in Table 1 and Table 2. Also, we have compared in Figure 1 - Figure 2 the numerical values of $f$ and $f^{\prime}$ with result of LTNHPM and also $\theta(\eta)$ and $\omega(\eta)$ with different values of Prandtl and Schmidt numbers in Figure 3 - Figure 5. The results are found to be in good agreement. The results show that the LTNHPM is an effective mathematical tool which can play a very important role in nonlinear sciences.

\section{Appendix A. Nomenclature}

$v \quad$ velocity component in the $y$ direction

HPM homotopy perturbation method

NM numerical method

$D_{A B} \quad$ diffusivity

$C_{p} \quad$ Specific heat capacity

$P \quad$ pressure

$p \quad$ parameter of homotopy

Pr Prandtl number 
Hossein Aminikhah, Ali Jamalian / TJ MCS Vol .5 No.4 (2012) 241-257

$\begin{array}{ll}S c & \text { Schmidt number } \\ R e & \text { Reynolds number } \\ T & \text { temperature } \\ T_{W} & \text { temperature imposed on the plate } \\ T_{\infty} & \text { local ambient temperature } \\ u & \text { velocity component in the } x \text { direction } \\ x & \text { dimensional vertical coordinate } \\ y & \text { dimensional horizontal coordinate } \\ \omega_{A} & \text { Mass fraction of species A } \\ \omega_{A} & \text { Mass fraction of species A on the plate } \\ \omega_{A \infty} & \text { Local ambient mass fraction of species A } \\ \text { Greek symbols } & \text { density } \\ \rho & \text { volumetric thermal expansion coefficient } \\ \beta & \text { kinematic viscosity } \\ v & \text { thermal diffusivity } \\ \alpha & \text { dimensionless temperature } \\ \theta & \text { dimensionless mass concentration } \\ \omega & \end{array}$

\section{References}

[1] H. Blasius The Boundary Layers in Fluid with Little Friction (in German) Zeitschrift fur Mathematik und Physik, 56 (1) 908 1-37; English translation available as NACATM 1256, February 1950.

[2] J.H. He, Homotopy perturbation technique, Comput Meth Appl Mech Eng,178, (1999) 257-262.

[3] J.H. He, A coupling method of homotopy technique and perturbation technique for nonlinear problems, Int J Non-linear Mech, 35, (2000) 37-43.

[4] J.H. He, New interpretation of homotopy perturbation method, Int J Mod Phys B, 20, (2006) 2561-8.

[5] J.H. He, Recent development of homotopy perturbation method, Topol. Meth Nonlinear Anal,31, (2008) 205-9.

[6] J.H. He, The homotopy perturbation method for nonlinear oscillators with discontinuities, Appl Math Comput,151, (2004) 287-92.

[7] J.H. He, Application of homotopy perturbation method to nonlinear wave equations, Chaos Soliton Fract,26, (2005) 695-700.

[8] J.H. He, Limit cycle and bifurcation of nonlinear problems, Chaos Soliton Fract,26, (2005) 82733. 
Hossein Aminikhah, Ali Jamalian / TJ MCS Vol .5 No.4 (2012) 241-257

[9] A. Rajabi, D.D. Ganji, Application of homotopy perturbation method in nonlinear heat conduction and convection equations, Phys Lett A,360, (2007) 570-3.

[10] D.D. Ganji, A. Sadighi, Application of homotopy perturbation and variational iteration methods to nonlinear heat transfer and porous media equations, J Comput Appl Math, 207, (2007) 2434.

[11]D.D. Ganji, The application of Hes homotopy perturbation method to nonlinear equations arising in heat transfer, Phys Lett A, 355, (2006) 337-41.

[12] G.A. Afrouzi, D. D. Ganji, H. Hosseinzadeh, R.A. Talarposhti, Fourth order Volterra integro differential equations using modifed homotopy-perturbation method, The Journal of Mathematics and Computer Science, 3 (2011) 179-191.

[13] Mohamed I. A. Othman, A. M. S. Mahdy and R. M. Farouk, Numerical Solution of 12th Order Boundary Value Problems by Using Homotopy Perturbation Method, The Journal of Mathematics and Computer Science, 1 (2010) 14-27.

[14]J. Singh, D. Kumar, Sushila, S. Gupta, APPLICATION OF HOMOTOPY PERTURBATION TRANSFORM METHOD TO LINEAR AND NON-LINEAR SPACE-TIME FRACTIONAL REACTIONDIFFUSION EQUATIONS, The Journal of Mathematics and Computer Science, 5 (2012) 40-52.

[15]S. Abbasbandy, A numerical solution of Blasius equation by Adomians decomposition method and comparison with homotopy perturbation method, Chaos Soliton Fract,31, (2007) 257-60.

[16]J. Biazar, H. Ghazvini, Exact solutions for nonlinear Schrodinger equations by He's homo-topy perturbation method, Phys Lett A, 366, (2007) 79-84.

[17] S. Abbasbandy, Numerical solutions of the integral equations: homotopy perturbation and Adomians decomposition method, Appl Math Comput,173, (2006) 493-500.

[18]JH. He, Homotopy perturbation method for solving boundary value problems, Phys Lett A,350, (2006) 87-8.

[19] Q. Wang, Homotopy perturbation method for fractional KdV-Burgers equation, Chaos Soliton Fract,35, (2008) 843-850.

[20]E. Yusufoglu, Homotopy perturbation method for solving a nonlinear system of second order boundary value problems,Int J Nonlinear Sci Numer Simul, 8, (2007) 353-8.

[21] Y. Khan, N. Faraz, A. Yildirim and Q. Wu, A Series Solution of the Long Porous Slider, Tribology Transactions,54, 2, (2011) 187-191.

[22] M. Esmaeilpour, D.D. Ganji, Application of He's homotopy perturbation method to boundary layer flow and convection heat transfer over a flat plate, Physics Letters A 372, (2007) 33-38.

[23] L. Howarth, On the Solution of the Laminar Boundary-Layer Equations, Proceedings of the Royal Society of London, A 164 (1983) 547-579.

[24]H. Aminikhah, Analytical Approximation to the Solution of Nonlinear Blasius' Viscous Flow Equation by LTNHPM, ISRN Mathematical Analysis vol. 2012, Article ID 957473, 10 pages, 2012. doi:10.5402/2012/957473. 
Hossein Aminikhah, Ali Jamalian / TJ MCS Vol .5 No.4 (2012) 241-257

[25]H. Aminikhah, M. Hemmatnezhad, An efficient method for quadratic Riccati differential equation, Commun. Nonlinear Sci. Numer. Simul. 15 (2010) 835-839.

[26]H. Aminikhah, A. Jamalian, A new efficient method for solving the nonlinear Fokker-Planck equation, Scientia Iranica, In Press, Available online 3 July 2012.

[27]H. Aminikhah, F. Mehrdoust, A. Jamalian, A New Efficient Method for Nonlinear Fisher-Type Equations, Journal of Applied Mathematics, vol. 2012, Article ID 586454, 18 pages, 2012. doi:10.1155/2012/586454.

[28] R. Byron Bird, Warren E. Stewart, Edwin N. Lightfoot, Transport Phenomena, John Wiley\& Sons (ASIA) Pte Ltd, 627. 